\title{
Evaluation of Marked Rise in Fecal Egg Output after Bithionol Administration to Horse and its Application as a Diagnostic Marker for Equine Anoplocephala perfoliata Infection
}

\author{
Yoshinori SANADA ${ }^{1)}$, Hiroyuki SENBA ${ }^{2)}$, Rieko MOCHIZUKI ${ }^{3)}$, Hidemi ARAKAKI ${ }^{3)}$, Tadahiro GOTOH ${ }^{3)}$, \\ Shin-ichiro FUKUMOTO ${ }^{3)}$ and Hajime NAGAHATA ${ }^{4) *}$ \\ ${ }^{1)}$ Sanada Veterinary Clinic, 573-6 Utafue, Mitsuishi, Hokkaido 059-3351, ${ }^{2)}$ Livestock Clinical Center, Hidaka Agricultural Insurance
Association, 200 Mitsuishi Higashihorai, Shinhidaka-cho, Hidaka, Hokkaido 059-3105 and ${ }^{3)}$ Department of Parasitology,
${ }^{4}$ Animal Health, School of Veterinary Medicine, Rakuno Gakuen University, 582 Bunkyodai-Midori, Ebetsu, Hokkaido 069-8501, Japan
}

(Received 9 June 2008/Accepted 11 January 2009)

ABSTRACT. To establish a reliable diagnostic measure for equine Anoplocephala perfoliata infection, the impact of deworming was examined in 12 Thoroughbreds to which bithionol (5-10 mg/kg body weight) was administered and feces were examined by the modified Wisconsin method using sucrose solution. One day after the administration, cestode eggs were detected in previously fecal egg-negative 3 horses and increased in the other 9 horses. The optimum time for post-deworming egg detection was examined in following horses: 17 mares were administered bithionol and 10 mares were used as controls. The fecal egg count was significantly $(\mathrm{P}<0.01)$ higher one day after the administration than that on other pre- and post-administration days, while no significant changes occurred in fecal egg count in the controls, demonstrating that one day after bithionol administration is the optimum time for detecting fecal cestode eggs. The diagnostic deworming involving bithionol and fecal examination on the day following administration provides a reliable diagnosis for equine Anoplocephala perfoliata infection.

KEY WORDS: Anoplocephala perfoliata, bithionol, diagnostic method, egg count, horse.

J. Vet. Med. Sci. 71(5): 617-620, 2009

Infection of horses by endoparasites has traditionally been a major issue in Thoroughbred production areas in Japan [18, 22]. Three tapeworm species, Anoplocephala perfoliata, Anoplocephala magna, and Paranoplocephala mamillana, have been reported in horses in the world $[1,6$, $9,14,19]$. Of those, Anoplocephala perfoliata is the most common and most pathogenic species infecting horses worldwide including Japan [1, 6, 13, 14, 17, 21]. Paranoplocephala mammilana is very rare, and Anoplocephala magna has been detected only in non-Thoroughbred varieties in Japan [1, 2, 13, 17-19]. Thus, Anoplocephala perfoliata is the most dominant cestode in Thoroughbred horses in Japan.

It has been thought that Anoplocephala perfoliata infection is subclinical and causes no severe clinical conditions. Upon autopsy, however, a large number of tapeworms are found concentrated at the ileocecal junction and cecum of horses suffering ruptured cecum $[7,10,13,19]$. When associated with volvulus or ruptured cecum, the infection is thought to result in lethal outcomes [10, 19].

Hearn et al. [5] reported that a significantly larger number of eggs of tapeworms were observed in fecal samples collected $24 \mathrm{hr}$ after administration of an anti-parasitic, pyrantel pamoate. Our previous study also showed that administration of pyrantel pamoate resulted in detection of Anoplocephala perfoliata parasites in feces of 58.8\% of 107

\footnotetext{
* Correspondence to: Dr. Nagahata, H., School of Veterinary Medicine, Rakuno Gakuen University Ebetsu, Hokkaido 0698501, Japan.

e-mail : nagahata@rakuno.ac.jp
}

horses, a marked contrast to the egg positive rate of $27.5 \%$ before the administration [13]. In order to utilize this "diagnostic deworming" in establishing a more reliable Anoplocephala perfoliata egg detection method, the impact of diagnostic deworming and the optimum time for detecting fecal Anoplocephala perfoliata eggs should be determined.

The aim of this study was to explore the impact of deworming on egg detection and to determine the optimum time for detecting Anoplocephala perfoliata eggs in horse feces after deworming. For deworming, we used bithionol, an anti-tapeworm drug that has been proven effective against Anoplocephala perfoliata [2] and is currently provided in an easy-to-use paste form $[17,18]$.

\section{MATERIALS AND METHODS}

Horses: Forced oral administration of bithionol (5 to 10 $\mathrm{mg} / \mathrm{kg}$ body weight; SILNAC ${ }^{\circledR}$ Paste; Dainippon Pharmaceuticals Co., Ltd., Tokyo) was carried out in a total of 12 clinically healthy Thoroughbreds (7 mares and 5 foals) of a breeding farm in Hidaka District, Hokkaido.

Collection of feces: Fresh feces were collected into nylon gloves on the day of bithionol administration (Day 0), the day following the administration (Day 1) and 10 days after the administration (Day 10). The feces samples were kept at 4C and used within 7 days after collection.

Cestode egg counts: Ten g of fresh feces were treated according to the procedure as described [13]. Cestode eggs were counted by the modified Wisconsin method [11, 20] using sucrose solution, and EPG was calculated. 
Field trial of diagnostic deworming: Twenty-seven clinically healthy Thoroughbred mares of 4 to 17 years of age from 3 farms (A through C) in Hidaka District were used. Horses in each farm were allocated to Administration and Control Groups (Farm A, 7 and 3 horses; Farm B, 5 and 2 horses; and Farm C, 5 and 5 horses, respectively), totaling 17 horses in the Administration Group and 10 horses in the Control Group. Bithionol (SILNAC ${ }^{\circledR}$ Paste; Dainippon Pharmaceuticals Co., Ltd., Tokyo) was administered once at a dose of 5 to $10 \mathrm{mg} / \mathrm{kg}$ body weight to the Administration Group. Fresh feces were collected 7 days before the administration (Day -7), on the day of administration (Day 0), and 1, 2 and 7 days after administration (Day 1, 2 and 7, respectively) and refrigerated at $4^{\circ} \mathrm{C}$ until testing. EPG was determined by the modified Wisconsin method using sucrose solution.

Statistics: Two-by-two contingency table was prepared for the individuals in both groups whose egg counts increased or decreased $24 \mathrm{hr}$ before/after administration of bithionol, and the results were assessed by chi-square test. The geometric average was obtained for each sampling date for each group and was compared between the groups by the Wilcoxon signed rank test. Values of $\mathrm{p}<0.05$ were regarded significant.

\section{RESULTS}

We conducted a preliminary trial to determine the impact of deworming on the efficiency of cestode egg detection. Bithionol was administered to 7 mares and 5 foals, and the number of fecal cestode eggs was monitored by the modi- fied Wisconsin method using sucrose solution (Table 1). On Day 1 after the administration, the number of fecal cestode eggs was increased in all 12 horses, including the 3 horses that were negative for fecal eggs on Day 0. On Day 10, only one horse remained positive for fecal cestode eggs; this horse was negative on Day 0 and turned positive on Day 1. Since the EPG values were not in normal distribution, geometric mean was calculated for each sampling date for statistic analysis. The EPG for Day 1 was significantly higher than that for Day 0 or Day $10(\mathrm{p}<0.01$ and $\mathrm{p}<0.001$, respectively) (Table 1).

A field trial of diagnostic deworming was conducted to determine the optimum time for fecal egg detection. The number of fecal cestode eggs was determined on Day -7, 0, 1,2 , and 7 in 17 horses which were administered bithionol and 10 control horses without administration (Table 2). In the Administered Group, all of the 17 horses were found positive for fecal Anoplocephala perfoliata eggs on Day 1 after the administration, as opposed to 16 positives found on Day -7 before the administration. On Day 1, the fecal egg count increased 13 to 19-fold from the values on Day -7 and was significantly higher than those for other sampling days $(\mathrm{P}<0.001)$. The fecal egg count decreased rapidly by Day 2 and, on Day 7, 11 horses of the Administered Group had an EPG of 0 . The EPG for Day 7 was significantly lower than those for other sampling days $(\mathrm{p}<0.01)$. In contrast, no significant changes were observed in the fecal egg count in the Non-Administered Control Group on Day 1, 2 or 7, and 7 of the 10 control horses were positive for fecal cestode eggs on Day 7.

Table 1. Change of EPG value of Anoplocephala perfoliata in the preliminary deworming trial with bithionol

\begin{tabular}{llrc}
\hline No Horse & Day 0 & Day 1 & Day 10 \\
\hline 1 Mare & 1 & 5 & 0 \\
2 Mare & 4 & 78 & 0 \\
3 Mare & 1 & 71 & 0 \\
4 Mare & 5 & 9 & 0 \\
5 Mare & 6 & 35 & 0 \\
6 Mare & 1 & 8 & 0 \\
7 Mare & 0 & 6 & 0 \\
8 Foal & 0 & 16 & 0 \\
9 Foal & 0 & 3 & 1 \\
10 Foal & 10 & 151 & 0 \\
11 Foal & 2 & 12 & 0 \\
12 Foal & 2 & 7 & 0 \\
\hline Geometric mean & $* 1.7$ & 16.5 & $* 0.1$ \\
SD & 1.24 & 2.23 & 0.22 \\
Number of negative & 3 & 0 & 12 \\
Number of positive & 9 & 12 & 1 \\
Positive rate(\%) & 75.0 & 100.0 & 8.3 \\
\hline Number increased in EPG & & 16 & 0 \\
Number decreased in EPG & & 0 & 0 \\
Number of negative to positive & & 3 & 11 \\
Number of positive to negative & & 0 &
\end{tabular}

* Significantly fewer than the day $1 \quad(\mathrm{p}<0.01)$. 
Table 2. Changes in fecal egg output during diagnostic deworming with bithionol

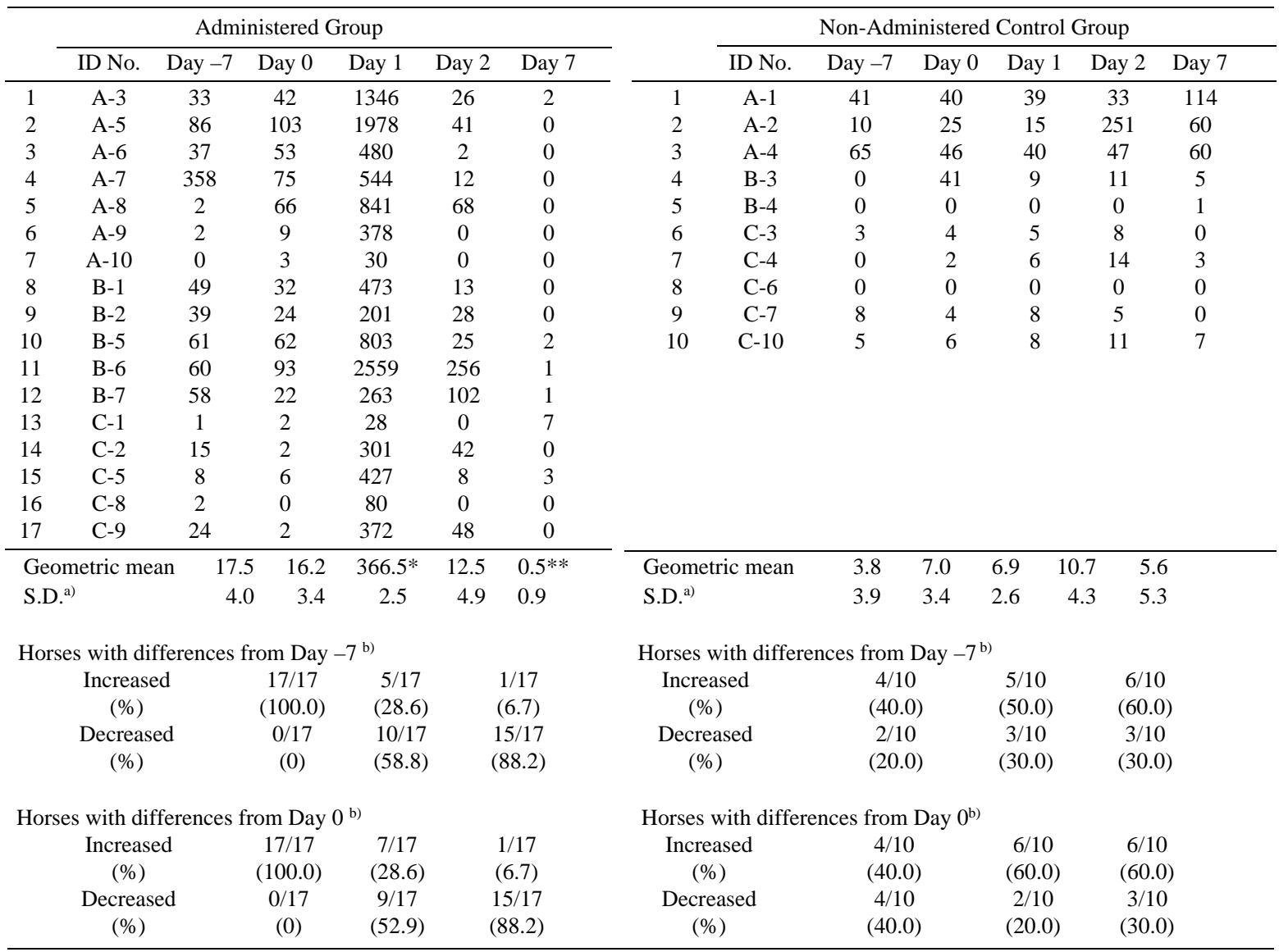

a) Standard deviation.

b) The number of horses with increased or decreased fecal egg output from that on Day -7 or 0/the total number of horses.

The values in parentheses are percentages.

*, $\mathrm{p}<0.001$ vs. other sampling days.

**, $\mathrm{p}<0.01$ vs. other sampling days

\section{DISCUSSION}

Our preliminary trial of diagnostic deworming demonstrated that bithionol markedly increased the fecal egg counts in all horses, which indicated a significant impact of bithionol administration on the reliability of fecal egg detection and provided impetus to a larger scale field trial to determine the optimum post-deworming time for egg detection. The field trial demonstrated that the fecal egg count in the Administered Group increased markedly on the day following the administration compared to the pre-deworming values, indicating that diagnostic deworming increased the reliability of detecting cestode eggs in feces. The fecal egg count decreased sharply by Day 2 after the administration, and 11 horses (64.7\%) were negative on Day 7 for fecal cestode eggs. No such changes were observed in the NonAdministered Control Group. These findings indicate that the day following the administration of bithionol is optimum for detecting fecal Anoplocephala perfoliata eggs.

Our present data are in agreement with previous studies on the beneficial effect of deworming upon fecal cestode egg detection. Hearn and Hearn [5] showed that fecal samples collected after administration of pyrantel pamoate, a drug that acts upon neuromuscular junctions and causes paralysis, provide a better indication of tapeworm infection than pretreatment samples. A study on the efficacy of pyrantel pamoate reported that fecal cestode egg count peaked 18 to $24 \mathrm{hr}$ after the administration with a value approximately 10 times the pre-administration value [15]. Similarly, administration of praziquantel, which disturbs membrane potential of muscle cells and paralyzes parasites, was followed by a significant increase in fecal egg count 18 to $24 \mathrm{hr}$ after the administration [16]. All these studies used the Cornell-Wisconsin centrifugal flotation method for fecal egg count $[15,16]$. Thus, it appears agreeable that deworming helps improve the reliability of fecal egg detection method, although the optimum time for fecal examination may vary with the used anthelmintics.

Bithionol is a known uncoupler of oxidative phosphorylation that inhibits ATP synthesis [4]. When exposed to 
bithionol, Anoplocephala perfoliata parasites have their ATP synthesis inhibited, detach from the intestinal wall and are excreted along with feces in the form of proglottide. When intact, Proglottides are so robust that they do not succumb to the pressure of pinching fingers. However, as their metabolic activities are further compromised by bithionol, proglottides degenerate and release eggs, resulting in increased fecal egg output. A study on the efficacy of bithionol reported that excreted tapeworms were most abundant one or two days after bithionol administration [13, 17]. This finding accounts in part for our present findings that fecal egg output peaked on Day 1 after the bithionol administration, because fecal samples for coprological egg detection are crushed in a mortar, which would result in physical destruction of excreted parasites and release of their eggs.

Given the prevalence of equine Anoplocephala perfoliata infection ranging from $13 \%$ to $82 \%[5,6,8,9,11,13]$ and the fact that a large majority of grazing foals is infected [4], it is of critical importance for horse farms to apply the diagnostic deworming that we described in the present study to a fraction of herd and, when necessary, to conduct herd-wide deworming. The present study showed that bithionol administration prior to fecal examination is effective in improving the reliability of fecal egg detection. The present approach is sufficiently effective and practical to survey for the prevalence of equine Anoplocephala perfoliata in horses.

ACKNOWLEDGMENTS. We would like to thank Dainippon Pharmaceutical Co., Ltd. for providing SILNACQ ${ }^{\circledR}$ Paste for this study. The assistance in fecal examination by the members of the Department of Veterinary Parasitology, Rakuno Gakuen University, was invaluable. We are indebted to Dr. Toshio Homma for critical reading of the manuscript. This work was supported in part by grants from Rakuno Gakuen University Academic Frontier and Joint Research (1993 and 2000 to 2006).

\section{REFERENCES}

1. Fukui, M. 1960. Studies on equine tapeworms and their intermediate hosts (1) Studies on the incidence of equine tapewoms, Anoplocephala perfoliata Goeze, 1782 and Anoplocephala magna Abildgaard, 1789 and the experimental studies on the removal of these cestodes with bithionol. Jpn. J. Parasitol. 9: 190-194 (in Japanese with English title and summary).

2. Fukui, M., Kaneko, C. and Ogawa, A. 1960. Studies on equine tapeworms and their intermediate hosts (2) Studies on removal effect of bithionol, bithionol acetate and dichlorophen for equine tapeworms, Anoplocephala perfoliata. Jpn. J. Parasitol. 9: 217-223 (in Japanese with English title and summary).

3. Gasser, R.B., Williamson, R.M. and Beveridge, I. 2005. Anoplocephala perfoliata of horses-significant scope for further research, improved diagnosis and control. Parasitology 131: 113.

4. Hamajima, F. 1973. Studies on metabolism of lung fluke genus Paragonimus. VII. Action of bithionol on glycolytic and oxida- tive metabolism of adult worms. Exp. Parasitol. 34: 1-11.

5. Hearn, F.P.D. and Hearn, E.E. 1995. A simple diagnostic technique to better determine the prevalence of tapeworms. $J$. Equine Vet. Sci. 15: 96-98.

6. Hoglund, J. 1998. Epidemiology of Anoplocephala perfoliata infection in foals on a stud farm in south-western Sweden. Vet. Pathol. 75: 71-79.

7. Ihler, C.F., Rootwelt, V., Heyeraas, A. and Dolvik, NI. 1995. The prevalence and epidemiology of Anoplocephala perfoliata in Norway. Vet. Res. Commun. 19: 487-494.

8. Lyons, E.T., Drudge, J.H., Tollivar, S.C. and Swerczek, T.W. 1986. Pyrantel pamoate: Evaluating its activity against equine tapeworms. Vet. Med. 813: 280-285.

9. Nilsson, O., Ljungstrom, B.L., Hoglund, H., Lundquist, H. and Uggla, A. 1995. Anoplocephala perfoliata in horses in Sweden: prevalence, infection levels and intestinal lesions. Acta Vet. Scand. 36: 319-328.

10. Pearson, G.R., Davies, L.W. and White, A.L. 1993. Pathological legions associated with Anoplocephala perfoliata at the ileo-caecal junction of horses. Vet. Rec. 132: 179-182.

11. Proudman, C.J. and Edwards, G.B. 1992. Validiation of a centrifugation/floatation technique for the diagnosis of equine cestodiasis. Vet. Rec. 131: 71-72.

12. Proudman, C.J. and Edwards, G.B. 1993. Are tapeworms associated with equine colic? A case of control study. Equine Vet. J. 25: 224-226.

13. Sanada, Y., Asano, H., Ohishi, H. and Senba, H. 1998. Relation between the positive rate of egg count and the infection rate of Anoplocephala perfoliata in horses. J. Jpn. Vet. Med. Assoc. 51: 495-498 (in Japanese with English title and summary).

14. Slocombe, J.O.D. 1979. Prevalence and treatment of tapeworms in horses. Can. Vet. J. 20: 136-140.

15. Slocombe, J.O. 2004. A modified critical test for the efficacy of pyrantel pamoate for Anoplocephala perfoliata in equids. Can. J. Vet. Res. 68: 112-117.

16. Slocombe, J.O. 2006. A modified critical test and its use in two dose titration to assess efficacy of praziquantel for Anoplocephala perfoliata in equids. Vet. Parasitol. 136: 127-135.

17. Toguchi, M., Yoshihara, T. and Otake, K. 2004. Evaluation of anthelmintic efficacy of bithionol paste against tapeworms naturally infected in horses, by fecal examination and necropsy; a critical trial. J. Equine Sci. 15: 37-41.

18. Toguchi, M. and Chinone, S. 2005. Helminthological survey of race horses on the basis of fecal examination. J. Jpn. Vet. Med. Assoc. 58: 247-249 (in Japanese with English title and summary).

19. Uhlinger, C.A. and DiPietro, J. 2002. Equine tapeworm infection. pp.1445-1447. In: Large Animal Internal Medicine, 3rd ed. (Smith, B.P. ed.), Mosby, St.Louis.

20. Williamson, R. M., Beveridge, I. and Gasser, R. B. 1998. Coprological methods for the diagnosis of Anoplocephala perfoliata infection of the horse. Aust. Vet. J. 76: 618-621.

21. Williamson, R.M., Gasser, R.B., Middleton, D. and Beveridge, I. 1997. The distribution of Anoplocephala perfoliata in the intestine of the horse and associated pathological changes. Vet. Parasitol. 73: 225-241.

22. Yoshihara, T., Oikawa, M., Hasegawa, M., Katayama, Y. and Kaneko, M. 1994. Prevalence of some internal parasites recovered at necropsy from race horses in Japan. J. Equine Sci. 5: 49-52. 